\title{
Ritual Music of the Kayapó-Xikrin, Brazil
}

Enregistrements (1995) de Max Peter Baumann

La musique rituelle des Kayapó-Xikrin, Brésil

\section{Riccardo Canzio}

\section{(2) OpenEdition}

\section{Journals}

Édition électronique

URL : http://journals.openedition.org/ethnomusicologie/1306

ISSN : 2235-7688

Éditeur

ADEM - Ateliers d'ethnomusicologie

\section{Édition imprimée}

Date de publication : 1 décembre 1996

Pagination : 350-352

ISBN : 978-2-8257-0559-9

ISSN : $1662-372 X$

\section{Référence électronique}

Riccardo Canzio, «Ritual Music of the Kayapó-Xikrin, Brazil », Cahiers d'ethnomusicologie [En ligne],

9 | 1996, mis en ligne le 05 janvier 2012, consulté le 20 avril 2019. URL : http://

journals.openedition.org/ethnomusicologie/1306

Ce document a été généré automatiquement le 20 avril 2019

Tous droits réservés 


\title{
Ritual Music of the Kayapó-Xikrin, Brazil
}

\author{
Enregistrements (1995) de Max Peter Baumann \\ La musique rituelle des Kayapó-Xikrin, Brésil
}

Riccardo Canzio

\section{RÉFÉRENCE}

Ritual Music of the Kayapó-Xikrin, Brazil.La musique rituelle des Kayapó-Xikrin, Brésil). CD SF 40433. Enregistrements (1995) de Max Peter Baumann (Smithsonian/Folkways Recordings - International Institute for Traditional Music). Notes de Lux Boelitz Vidal et d'Isabelle Vidal Giannini.

1 Les Kayapó, «peuple de la grande eau» (mebengôkre), vivent dans le Brésil central; on peut en trouver plusieurs groupes entre les fleuves Xingu et Tocantins. Les enregistrements furent effectués auprès des Xikrin de Cateté, un des sous-groupes kayapó vivant près du Tocantins. Comme la majorité des peuples autochtones, les Kayapó ont eu leur part de luttes dans les contacts avec la société qui les entoure, mais les deux mille cinq cents individus qui survivent semblent avoir mieux résisté aux ravages de la civilisation. Cela peut paraître exagéré mais on ne dira jamais assez combien la situation des Amérindiens est critique. Ce disque consacré aux rituels d'une culture peu connue présente l'aspect sonore d'une cérémonie d'attribution de nom qui est l'événement central de la vie rituelle des Kayapó. Dans des sociétés de ce type la musique peut être envisagée comme une activité créatrice qui régénère les relations sociales, renforce la cohésion du groupe et renouvelle le sens de l'identité culturelle.

La qualité des enregistrements numériques effectués sur le terrain est excellente. Compte tenu des difficultés inhérentes à ce type de travail, la prise de son est exemplaire et témoigne de ce que peut donner une utilisation intelligente de la technologie existante. 
3 La présentation graphique du texte d'accompagnement est impeccable. Deux magnifiques photos en couleur ornent la couverture et le dos de la plaquette; on aurait cependant aimé plus de photos, même en noir et blanc, pour illustrer ces rituels.

4 Les explications ethnographiques sont d'une qualité inhabituelle. Elles nous offrent soixante-quinze pages de textes, des graphiques ainsi que la traduction de certaines des paroles de la cérémonie, le tout suivi d'une brève bibliographie. On y trouve les titres de plusieurs ouvrages de Lux Vidal qui a consacré une partie de sa carrière d'anthropologue à l'étude de ce groupe. La langue, l'histoire, les différents aspects de la culture matérielle, le style de vie, l'organisation sociale et les mythes sont présentés avec talent et une réelle maîtrise. Quant aux détails ethnographiques de la cérémonie enregistrée, ils sont expliqués avec clarté et discernement.

5 Cependant, malgré la qualité irréprochable et la grande valeur documentaire de ces explications, on aurait souhaité un peu plus d'informations sur le système musical luimême: des données sur la fonction de la musique et sur sa pratique par ce groupe ethnique ainsi qu'une évaluation des formes musicales; peut-être également une classification des chants et un glossaire des termes musicaux en langue kayapó. En un mot, quelques réflexions sur la musique et sur les aspects sonores de pratiques rituelles, qui, d'habitude, ne retiennent pas l'attention des anthropologues.

6 Ayant moi-même travaillé avec des groupes ethniques d'Amazonie, je suis parfaitement conscient des difficultés qui se présentent lorsqu'on recueille ce genre de données et je sais qu'une musique de ce type, profondément enracinée dans son substrat socioculturel, présente un grand problème de description pour l'ethnomusicologue. Les instruments conceptuels dont il dispose ne sont pas encore parfaitement aiguisés.

7 En écoutant les chants, il semble que les paroles jouent un rôle important dans le choix de la forme musicale, et qu'elles constituent même la base sur laquelle s'élabore le contour mélodique qui les véhicule. Nous regrettons l'absence de transcriptions musicales, mais ce regret est en grande partie compensé par la transcription et la traduction d'une partie importante des paroles qu'on peut entendre dans ce disque. Il ne faut toutefois pas oublier que les enregistrements sonores ne donnent accès qu'à un seul paramètre de l'événement total: une cérémonie d'initiation et non un événement uniquement musical. Il ne s'agit pas d'une musique conçue dans un but purement esthétique mais plutôt des aspects musicaux d'un événement très complexe. On a affaire à un genre de musique dont l'exécution a tout autant d'importance que le résultat sonore.

8 On peut déplorer le fait que la notice n'ait pas cherché à relier plus étroitement les aspects ethnologiques et musicaux de ce travail, bien que ce genre de critique s'appliquerait plutôt à un ouvrage ethnomusicologique au plein sens du terme qu'à un excellent document tel que celui-là. Il s'agit d'une incursion dans le terrain qui vient au bon moment et qui fera sans aucun doute gagner beaucoup de temps aux futurs musicologues en leur fournissant un matériau de première qualité. Il offre un complément utile au $\mathrm{CD}$ publié il y a cinq ans, avec des enregistrements des années soixante et soixante-dix, illustrant d'autres aspects de la musique des Xikrin ainsi que celle d'un groupe rattaché, les Kayapó-Mekrãgnoti (VDE 554/555, dont les Cahiers (4/1991: 293-296) ont publié un compte rendu).

9 Comme l'anthropologue, l'ethnomusicologue s'occupe, entre autres choses, de traiter du matériel ethnographique et de le présenter dans un discours cohérent où toutes les voix (les chercheurs précédents, les autochtones et l'auteur) ont leur place légitime; 
l'interprétation qui en découle naturellement propose une nouvelle vision, celle de l'auteur. Pour ce qui est de l'ethnomusicologie on devrait inclure les points de vue particuliers concernant la production musicale d'une société en essayant de les rattacher aux concepts autochtones. Tel est, d'après moi, la façon dont devraient procéder les futures recherches ethnomusicologiques dans ce domaine. L'étude des sociétés amérindiennes a énormément à apporter dans la réflexion théorique de cette discipline.

Nous avons dans ce disque une foule d'informations nous permettant de comprendre la vie, les actions et les sentiments d'un peuple amazonien qui reste fidèle à lui-même, bien qu'entouré d'une société pas toujours amicale. Nous avons aussi un bon exemple de ce que peut donner un enregistrement lorsqu'il est accompagné d'une notice intelligente. Tant par la clarté des idées exprimées que par la façon remarquable dont le travail est présenté, ce disque constitue pour moi une heureuse initiative.

11 Avec des enregistrements de ce genre, il y toujours une certaine ambiguité en ce qui concerne les droits d'auteur et des complications légales liées à leur payement. Dans ce cas précis, conformément à la politique instaurée par Smithsonian/ Folkways, les droits d'auteur reviennent à la communauté Xikrin où ont été faits les enregistrements. C'est un arrangement judicieux du point de vue éthique qui devrait être pratique courante bien que malheureusement ce soit rarement le cas.

12 Une telle publication est bienvenue dans un monde en principe concerné par les causes humanitaires et qui ne manque pas, pour la forme, de faire allusion aux valeurs de ceux dont le style de vie est différent, tout en ayant recours à des décisions politiques qui, en pratique, rejettent ces valeurs dans une série frénétique et irresponsable de mesures à court terme.

13 Ce disque est indispensable aux ethnomusicologues, anthropologues et toute personne intéressée par cette région. Nous le conseillons à ceux qui défendent les droits de l'homme et à tous ceux qui ont l'esprit curieux ou qui, tout simplement, sont concernés par les formes de culture et les modes de vie en péril.

\section{AUTEUR}

RICCARDO CANZIO

fr 